\title{
Do-it-yourself science
}

Alan Glynn

Who Goes First? The Story of Self-Experimentation in Medicine.

By Lawrence K. Altman. Random House: 1987. Pp. 430. \$22.50.

As Dr Johnson said in another context, "It is not done well; but you are surprised to find it done at all". One gets the same feeling, at any rate to start with, on reading some of the experiments that people have carried out on themselves. Altman's contribution is to show us not just that a few famous experiments have been of great scientific value but how extensive the whole process of self-experimentation is and. indeed. how necessary from an ethical point of view. If you are not prepared to experiment on yourself, how can you ask others?

Like all good books, this one can be read at several levels. At the most superficial it provides a good read for almost everyone. There is drama and mystery. What, for example, would Poirot have made of the case of the Canadian physiologist found alone and unconscious in the laboratory one Saturday evening? There are plenty of heroes and eccentrics, often combined. Castle who sorted out the intrinsic factor story in pernicious anaemia, was also a handyman and it was sometimes easier to consult one of the most illustrious professors at Harvard by asking him to mend a tap than to see a patient. On the other hand there are no real villains and no sex except for a few worried wives in the background. Indeed there are very few women in the book at all. Altman suggests this is a reflection of the relatively small number of women in medicine or medical research until recently. He is probably right (though there is also the possibility that women have more common sense than men). It is interesting but perhaps not significant that the woman who gets the most mention, Dr Elsie Widdowson, was a nutritionist. Mrs Goldberger insisted on sharing her husband's selfexperimentation into the causes of pellagra in order to represent women.

Nutrition, drugs, physiology and infection are the main fields covered, with the latter by far the largest. The risks and discomforts of each differ. Santorio of Padua spent 30 years of his life with bed and table on a steelyard to measure the weight changes due to what we would now call metabolism; tedious but hardly dangerous. Others were not so lucky. In 1769 William Stark tried to see if simple diets were healthy, and died of scurvy, though James Lind had described the remedy 16 years before.

Serendipity played its part. While investigating the properties of cocaine, Freud noticed a numbness of the tongue. $\mathrm{He}$ mentioned it to his friend, the ophthalmologist Koller, who developed

ophthalmologist Koller, who developed

Pettenkofer, though fortunate in only getting a mild diarrhoea after drinking a beakerful of cholera vibrios, was unlucky, because he did not recognize his illness and so was confirmed in his view that the micro-organism alone is insufficient. There is some truth in this, but unfortunately he went further and assumed he had demonstrated the need for a theoretical soil factor, in which he passionately believed. Of course he was anxious to prove a point, but such an approach is not confined to selfexperimenters. Complete scientific detachment is rare, though the courage to test scientific theories is clearly not.

Dr Altman traces back his interest in self-experimentation nearly 30 years and his qualities are reflected in several aspects of his book. As a physician with some experimental $\stackrel{\infty}{\circ}$ tendencies he gives an accurate พิ picture of the medical and scientific background to the $\sum_{2}^{\pi}$ experiments he describes, so allowing both himself and us to judge their proper worth. As a scholar he presents the detailed

छ results of his research in a field

where facts are difficult to come by. As a full-time journalist his writing is relaxed and interesting to read, with much human detail which hovers between verification of history from living sources and the journalistic interview. Just occasionally the latter takes over.

There are some interesting questions. What is the true story of John Hunter's autoinoculation? Why did Walter Reed go to Washington while his colleagues were being bitten by yellow-fever-bearing cted himself with a new AIDS vaccine. the use of cocaine as a local anaesthetic. Likewise, one day in Egypt Arthur Loos was surprised to find himself infected with hookworm, then thought to be spread only by ingestion. However, he remembered the burning sensation when a drop of a larval culture had fallen on his hand and went on to demonstrate experimentally the penetration of his intact skin by larvae of Ankylostomum duodenale.

There must be something about major advances. Loos's finding was not accepted for some time, because J.S. Haldane and Boycott could not at first confirm the results. This recalls the delay in the midnineteenth century in accepting Villemin's classical demonstration of the transmissibility of tuberculosis because an English Commission of distinguished scientists could not confirm his work. Or rather they did, but they managed also to infect one of the control guinea pigs, so coming to the wrong conclusions. mosquitoes? What were the

sensation motives of all these people? At a time when medicine is too often dismissed as technology, and anti-science - based as always on ignorance and superstition abounds, it is refreshing to read of men and women whose driving force was intellectual curiosity applied to problems of medicine no matter what the cost. To J.B.S. Haldane, the intellectual giant, it would have been an ideal way of dying. Pettenkofer and Metchnikoff among others, would have agreed. Dr Altman's book has itself gone first in exploring his subject. The questions he raises may be answered by some future student from the archives of the Institutions Review Boards and ethics committees, themselves inspired by the shadow of Nuremburg.

Alan Glynn is Director of the Central Public Health Laboratory, Colindale, London NW9 $5 H T, U K$, and a visiting Professor at the London School of Hygiene and Tropical Medicine, University of London. 